\title{
A Multiclass ELM Strategy in Pose-based 3D Human Motion Analysis
}

\author{
Arif Budiman \\ Computer Science Faculty, University of Indonesia \\ Email: arif.budiman21@ui.ac.id
}

\author{
Mohamad Ivan Fanany \\ Computer Science Faculty, University of Indonesia \\ Email: ivan.fanany@cs.ui.ac.id
}

\begin{abstract}
This paper pursues the best multiclass classification strategy for pose-based 3D human motion recognition using Extreme Learning Machines (ELM). Such classification task is one of the most difficult classification problem because the pose is not unique and the same pose might be randomly distributed inside any unrelated and absolutely different activities. In this study, bag of poses are selected as features and several multiclass classification strategies commonly used in binary classifiers such as Support Vector Machines (SVM) are adopted into the ELM implementation and then compared with non-binary classifications of ELM and binary classifications of SVM. A number of multiclass strategies such as One-Against-All (OAA), One-AgainstOne (OAO), Directed Acyclic Graph (DAG), hierarchical binary tree, and OAO-3Tree are evaluated and analysed. We found that the OAO-3Tree strategy using Max-Win vote fusion of labeled output function gives the best result.
\end{abstract}

\section{INTRODUCTION}

Human action recognition has received considerable attention from the computer vision community in many years with many progresses and remains an active area of research. It is the process of labeling image sequences with motion labels in semantic interpretation rather than intermediate-level (human tracking) or low-level vision (Human detection) [15]. Robust solutions to this problem have many applications in domains such as surveillance, human-computer interaction, interactive animation and digital choreography. The challenges in solving this problem, however, are multifold due to variations in motion performance, recording settings and inter-personal differences.

In this study, we analyse 3D human motions extracted from badminton sport actions to be categorized into 8 classes activity label of basic badminton sport actions. The input to the classifier are keyposes that is automatically extracted to represent a motion cluster or group. Compared to other action such as dance [17], sport action recognition is more difficult since there is no strict rules, rythms, or music to be followed by the actor during performance. This leads to large variations not only between different persons but also the same person performs in different time.

Pose-based 3D Human motion analysis in semantic level is categorized as a hard classification problem. Pose is primitive action like an element of movement at the limb level that possibly whole body locomotion or certain body part angular movement [11]. The difficulty of human motion analysis on semantic interpretation (high level vision) is due to the fact that the keypose in each motion cluster or action group is not unique and the same pose might be distributed anywhere to any different activities. Pose actions like jump, walk, swing, run and many others various actions might be repeatedly occurred on different location of sequence with different frequency of occurences in one activity label. In addition, more complex motions certainly need more number of keyposes and longer image sequences to adequately represent a richer motion variations belongs to multiclass of actions.

In efficiently dealing with such complex multiclass and long sequences of action, we need a fast and robust multiclass classification. In our previous research, we found that ELM eventhough fast but has low performance in posed-based 3D human motion analysis. We used better feature selection using bag of poses to improve the result without depending on the size of classifier structure. The problem that we are addressing now is how to improve better performance in multiclass settings. In this paper, we will compare various method of multiclass ELM classifier to solve posed-based 3D human motion classification problem. Our goal is to devise a better ELM multi classifier strategies in terms of recognition accuracy and optimum structure for faster processing time for a real-time video recognition. We proposed a number of multiclass ELM strategies such as One-Against-All (OAA), One-Against-One (OAO), Directed Acyclic Graph (DAG), hierarchical binary tree, and OAO3Tree. Those strategies were adopted from good reputation binary classifier support vector machine (SVM). Then we compared the result with the strategies using SVM multiclass classifier.

This paper is organized as follows. Section 1 gives introduction and objectives. Section 2 describes related works. Section 3 describes the pre-processing methods and Section 4 for performance verification and comparisons of ELM methods. Section 5 concludes this paper with discussions. 


\section{RELATED WORK}

In machine learning, classification task is how to identify a new observation as belongs to which set of categories, based on a training dataset which contains observations whose category of membership are already known. The learning process is supervised by labelled training set, the classifier will be able to categorize unknown objects into some known category classes. Classification design currently become the focus of intense research in machine learning communities. According to Kuncheva [8], she explained the taxonomy of methods for classifier design in Fig.1 even there is no consensus on a single taxonomy yet. Basically, it started with Bayes decision with the classconditional probability density function (pdf) and prior knowledge. However, in real-life problems, it is difficult to know the true prior probabilities nor the classconditional pdfs, we can only design by estimation function $\hat{P}\left(\omega_{i}\right)$ and $\hat{p}\left(\mathbf{x} \mid \omega_{i}\right)$ in the discriminant functions $g_{i}(\mathbf{x})=P\left(\omega_{i}\right) p\left(\boldsymbol{x} \mid \omega_{i}\right)$. Estimating pdfs is hard, especially in high dimensions with limited data. Another methods that are not derived by approximating the pdfs but rather by devising decision boundaries or discriminant functions empirically. Unfortunately, no dominant classifier exists for all the data distributions (no free lunch theorem), and the data distribution of the task at hand is usually unknown. That's why, instead of looking for the best set of features and the best classifier method, some researchers look for the best set of classifiers and the best combination method. Combining the opinions of an ensemble of pattern classifiers in the hope that the new opinion generated will be better than the individual ones.

Combining multi-classifiers now is state of the arts in pattern recognition research. According to Roli [2], multiple classifier systems (MCS) based on the combination of outputs of a set of different classifiers have been proposed as a method for the development of high performance classification systems. MCS methods can play an important role where decision-level fusion is required and not only improved the performance but also reliability. Roli [2] explained the motivation: 1) Traditional single classifier approach not works well when only small sample size available. For different choices of the training set, the best classifier varies. 2) Computational motivation. Many learning algorithms suffer from the problem of local minima even with enough training data. Fusion of multiple classifiers constructed by running the training algorithm from different starting points can better approximate the best classifier. MCS can be characterized by: 1) The Architecture/Topology. 2) The classifier Ensemble: type and number of base classifiers. The ensemble can be subdivided into subsets in the case of non parallel architectures. 3) The Fuser as decision function (see fig 2. The solution for multiclass classification problem is by decomposing into several binary classification tasks that can be solved efficiently using multiple binary

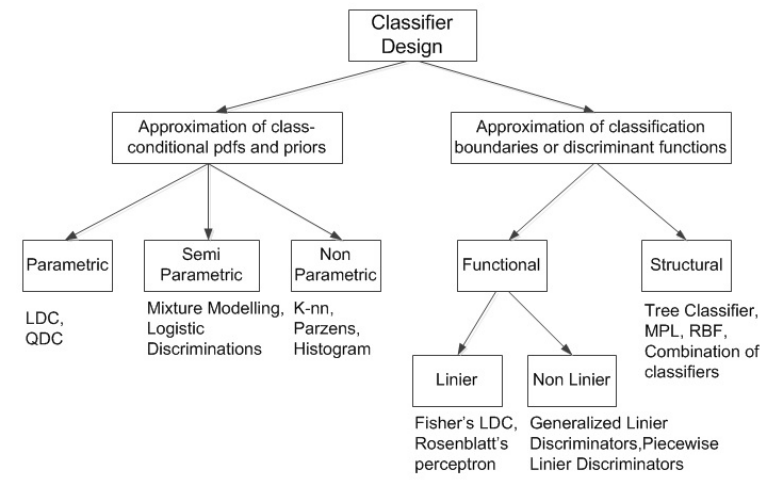

Fig. 1. A taxonomy of methods for classifier design

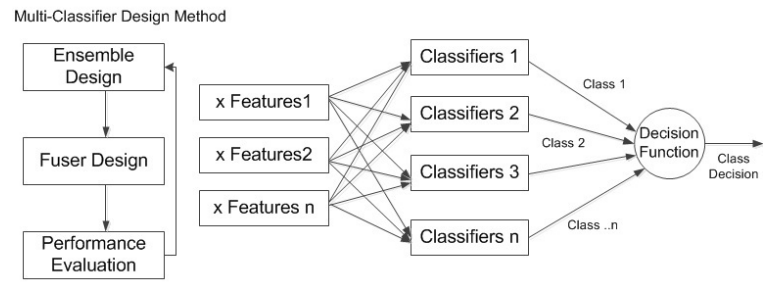

Fig. 2. Multi-Classifier Block Diagram

classifiers than single classifier approach.

SVM (Support Vector Machine) is consistently one of the top ten algorithms for data mining [16]. It is a state-of-the-art learning machine that is used in many areas, even it is originally binary classifier. An SVM training algorithm builds a model that assigns new examples into one category or the other. So that the examples of the separate categories are divided by a clear gap that is as wide as possible. In learning process, SVM constructs a set of hyperplanes in a highor infinite-dimensional space, which can be used for classification or regression. SVM is powerful because it can achieve global minima, good generalization using Structural Risk Minimization (SRM) and easy implementation using quadratic optimization problem to find the support vectors. But, SVM has difficulties to handle complex multi-class classification problem directly. Vapnik has proposed since the origin time of SVM with MCS method one-against-all (OAA) [14] and developed further by $\mathrm{Kre} \beta \mathrm{el}$ with one-against-one (OAO) or pairwise methods [13] and directed acyclic graph SVM (DAGSVM) [1], [6]. The comparison results were that pairwise SVMs (OAO) would be somewhat slower than OAA but produce more accurate results on the data set [1], [12], [13].

The conventional max-win-voting (MWV) method is commonly used as fuser decision function [8], [2]. Some papers were introducing a hierarchical architecture model like unbalanced decision tree (UDT) [10] and clustering algorithm to determine the hierarchy of binary decision subtasks [6]. Hierarchical model is basically using some binary classifiers in cascaded composition, so the decision making process contributed by each classifier in serial/parallel/both 
connection to the next level of classifier. Common example model is binary tree.

Later on, ELM was introduced and makes contradictory thinking of iteration learning process. The ELM uses simple learning algorithm for Single Hidden Layer Feedforward Network (SLFN). The algorithm can prove if weight input and bias in hidden layer initialized with random value then we can use any infinitely differentiable activation function to make SLFN become a linier system. ELM training process is explained below:

1) Currently, no known a priori standard to determine the number of hidden nodes. Generally, it is based on experiments and may vary depends on the training samples. Some experiments explained methods to get better accuracy is by adding the number of hidden nodes.

2) The weight input $W_{i}$ and bias $b_{i}$ can be assigned with random numbers matrix sized by Number of Hidden Neurons.

3) Hidden layer matrix $\mathbf{H}$ is like an information base of training data without a knowledge. $\mathbf{H}$ is calculated as non linear differentiable function of the summation matrix result from bias matrix $\mathrm{b}$ and the weight input $\mathrm{W}$. In matrix $\mathbf{H}$, there is still no information from supervised learning process.

4) The Output weight $\beta$, where $\beta=\mathbf{H}^{\dagger} \mathbf{T}$, which $\mathbf{H}^{\dagger}$ known as Moore Penrose generalized inverse (Pseudo Inverse) of H. Output Weight is like a knowledge base because it has a knowledge from supervised learning driven by target data matrix T.

5) During the testing, ELM will call the knowledge base Output Weight and multiplied it with the information base obtained from input data to get the result

Compared with traditional SVM and LS-SVM, Huang explained that ELM provides a unified learning platform can be applied in regression and multiclass classification applications directly. As verified by the simulation results, ELM tends to have better scalability and achieve similar (for regression and binary class cases) or much better (for multiclass cases) generalization performance at much faster learning speed [3]. Huang explained two methods: 1) Single ELM for the whole class number; The output nodes quantity will be equal to the total class number. For big data, this method will require more computation and longer training time. 2) Separated binary ELM for each class; Using OAO or OAA [4], [7]. In a single ELM classifier, the multi-class problem is implemented with an architecture of multi-output nodes which is equal to the number of pattern classes. Their performance is evaluated and showed that ELM-OAA and ELMOAO requires fewer hidden nodes than the single ELM classifier. In addition ELM-OAO usually has similar or less computation burden than the single ELM classifier when the pattern class labels is not larger than 10 [7]. In the ELM-OAA method, the m-class classification problem is implemented by m binary ELM classifiers, each of which is trained independently to classify one of the $\mathrm{m}$ pattern classes with the training data relevant to each class. The input data for each binary ELM classifier is the same but the target output data is different. For the qth binary ELM classifier, ELMq, the target output data need to be decomposed to two subsets: one labeled as 1 for all the qth class examples and the other labeled as -1 for all. In the ELM-OAO, the $\mathrm{m}$ pattern classes are pairwise decomposed into $\mathrm{m}(\mathrm{m}$ 1)/2 two different classes, each of which is trained by one ELM classifier $(\operatorname{ELMq}(j, s), q=1,2, \ldots$, $\mathrm{r} ;(\mathrm{r}=\mathrm{m}(\mathrm{m} \quad 1) / 2) ; \mathrm{j}=1,2, . ., \mathrm{m} \quad 1 ; \mathrm{s}=\mathrm{j}+1, \ldots, \mathrm{m})$ to discriminate two distinct classes. According to Rong [7], the comparison of testing accuracy of ELM-OAA, ELM and ELM-OAO achieve similar testing accuracy and thus it is hard to give a clear conclusion about their advantage on the testing accuracy (On image segmentation, handwritten and letter recognition case). However in our research with posed-based 3D Human motion analysis, we found the difference result in comparison experiments.

Some researchs showed that ELM training time is faster than SVM, but like SVM, ELM can avoid local minima [3], [4] because ELM training is simply equivalent to finding a least-squares solution $\hat{\beta}$ of the linear system $\mathbf{H} \beta=\mathbf{T}$ while hidden node parameters remaining fixed after randomly generated. Different with another learning methods, ELM aims at reaching the minimum training error :

$$
\begin{aligned}
& \left\|\mathbf{H}\left(w_{1}, \ldots, w_{\hat{N}}, b_{1}, \ldots, b_{\hat{N}}\right) \hat{\beta}-\mathbf{T}\right\| \\
& =\min _{\beta}\left\|\mathbf{H}\left(w_{1}, \ldots, w_{\hat{N}}, b_{1}, \ldots, b_{\hat{N}}\right) \beta-\mathbf{T}\right\| \\
& \text { (1) }
\end{aligned}
$$

as well as together with the smallest norm of the output weights that can be obtained from :

$$
\hat{\beta}=\mathbf{H}^{\dagger} \mathbf{T}
$$

\section{METHODS}

We collected the data using Kinect depth sensor camera positioned in front of the performer with distance 2-3 meters in closed area using openNI framework and Matlab. Kinect can generate skeleton information from human pose instantly. Skeleton joint is information map of joint coordinate in $3 \mathrm{D}(x, y, z)$. The joints are HEAD, NECK, LEFT SHOULDER, LEFT ELBOW, LEFT HAND, RIGHT SHOULDER, RIGHT ELBOW, RIGHT HAND, TORSO, LEFT HIP, LEFT KNEE, LEFT FOOT, RIGHT HIP, RIGHT KNEE, RIGHT FOOT (Total 15 Joints). We collected the Badminton data from 5 persons in different time and place for each motion activity classes are 
Long Service, Short Service,Overhead Loop, Forehand Stroke, Backhand Stroke, Drop Shot, Underhand lob, and Smash (Total 8 class).

Modifying [9], [17], skeleton joint features are represented by parameter of spherical 3D coordinate $\theta$ as inclination and $\varphi$ as azimuth. Except NECK (Due to small variance), we used $14 \theta$ and $14 \varphi$ joints position $\mathrm{x} N_{1 \ldots n}$ frame sequences (for each class). Using Kmeans clustering, all $28 \mathrm{x} \Sigma \mathrm{N}$ features clustered into a group of key poses from 5, 10, 15, 20, 25, 30, and 35 as key poses. Each motion class is represented on string symbol as a sequence of cluster pose ie. eeeeeeee, ddeeeeeeeeeee, etc. belong to the long service (class 1). The String symbol is converted to be numeric format and use only the frequency of pose occurence as feature (Bag of poses). The ELM input have to be normalized within range -1 to 1 , so the maximum value of feature is 1 and the minimum is -1 . We used sigmoid function and the hidden nodes number is various as multiplication from 1 to $5,10,20,50,100$ and 200 by key poses number.

Our previous study in the Pose-based 3D Human motion analysis using single ELM classifier only not giving the expected result. ELM structure needs to be grown up, and tends unstable in minimizing generalization error performance. However, SVM has success factor when using some MCS methods applied and the similar methods can be adopted for ELM to give better computation speed and accuracy. The methods are using decision tree topology with fixed rule using ELM as ensemble classifier and majority vote as fusion decision function for OAO and class selection method for OAA. Training data is splitted to be one against all (OAA) and pair wise one against one (OAO) methods. We compared ELM methods single classifier to multi classifer ELM with OAA, OAO, cascaded using binary tree and cascaded using OAO with 3tree (See figures 3-7). The idea of OAO with 3 tree is how to yield OAO performance with less classifier by grouping all class to be 3 training pair groups rather than $\mathrm{m}(\mathrm{m}-1) / 2$ pair groups. Then, the result of one group is purified by the next level of classifier until we get the membership class number.

There is possibility for no-class (class $=0$ ) conditional branch on multi classifier. On OAA class selection, no-class occured when any more than one classifier claimed the same class. On Max-Win vote, no-class occured when two classes voted by 3 classifiers (3-3-2). No-class is accumulated as false negative that affected to the accuracy performance. We can use no-class result to be re-classified by another MCS methods or assigned to random class number to give better overall result or solved it using DAG method. In DAG method, it is basically OAO method but using decision tree for classifiers to make decision (See fig 8).

To validate the result, we use cross validation with $\mathrm{K}$-fold with $\mathrm{K}=5$. To evaluate the quality, we use

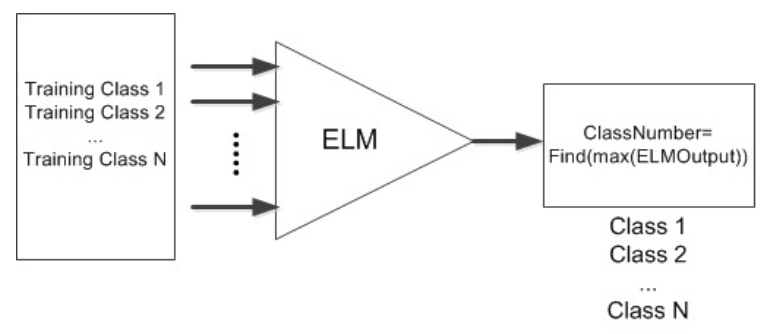

Fig. 3. ELM Single Classifier

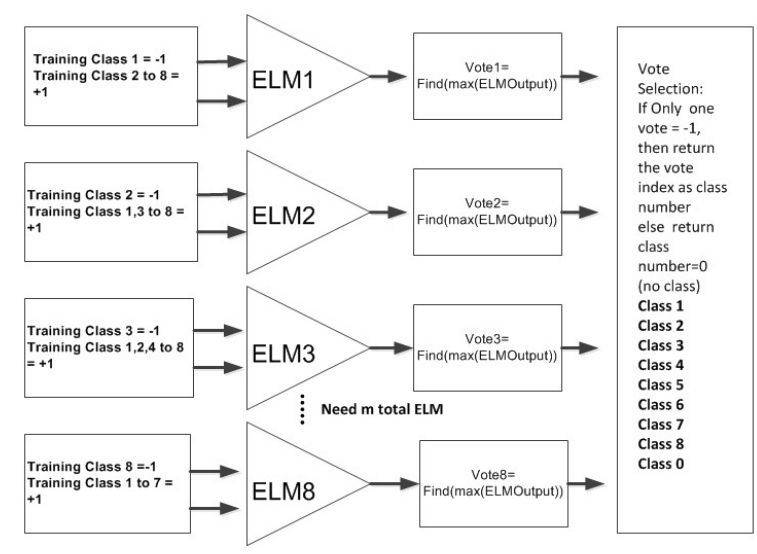

Fig. 4. ELM with OAA using 8 ELM Classifiers

accuracy, precision and recall with confusion matrix. Precision is a measure of exactness or quality, whereas recall is a measure of completeness or quantity. Accuracy is a measurement of how well a classification test correctly identifies. We used Matlab for all computation and code programming.

\section{Performance And Evaluations}

Based on our experiments, the best performance result in ELM is for ELM with OAO and DAG. However, ELM OAO needs 28 classifiers for 8 classes and become more complicated pair wise training model for more classes. It has good result for unbalanced data set in class3, class 7 and class 8 . Followed by OAO-3Tier with 10 classifiers and binary tree with only 7 classi-

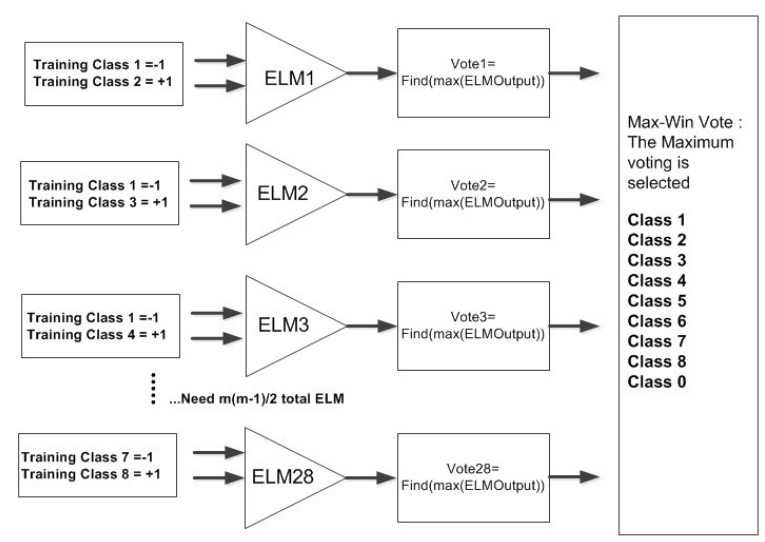

Fig. 5. ELM with OAO/Pairwise Method using 28 ELM Classifiers 


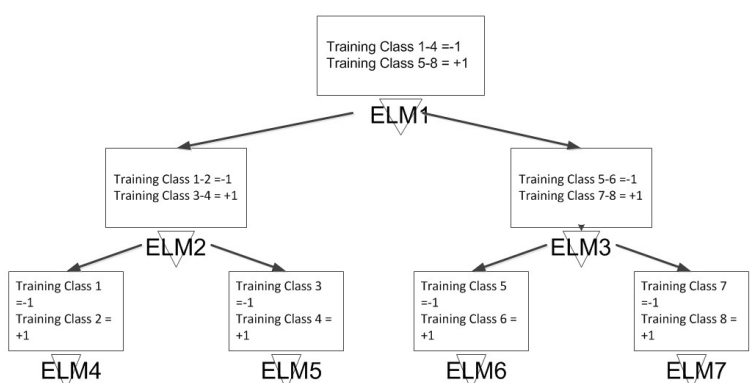

Fig. 6. ELM with Balanced data set-Binary Tree Hierarchy using 7 ELM Classifiers

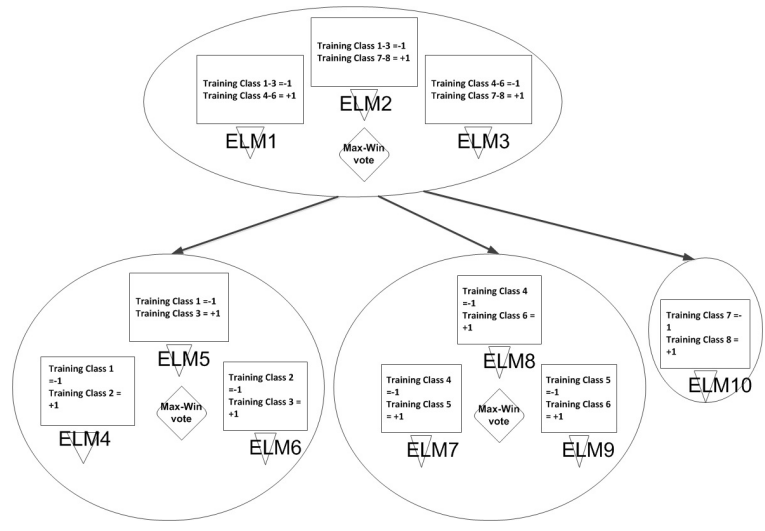

Fig. 7. ELM with OAO-3Tree Hierarchy using 10 ELM Classifiers

fiers. ELM OAA has relatively similar performance with single classifier because the selection method giving many no-class result. Class 3 , class 7 and class 8 improved better when we splitted to smaller class pairwise comparison. ELM OAO, ELM-DAG, binarytree and OAO-3tree methods are giving better stability for more increasing hidden nodes number (Fig. 9) and more scalability with improved performance for high pose cluster group (Fig. 10) than single classifier and OAA methods. However, the accuracy performance result is still not good as SVM method. SVM OAA and OAO can achive higher accuracy (except for pose cluster group=5) with slower training time and complex iteration process. We used Matlab version of SVM with quadratic kernel function, quadprog_opts and using 1000 maximum iteration to solve the SVM optimization problem.

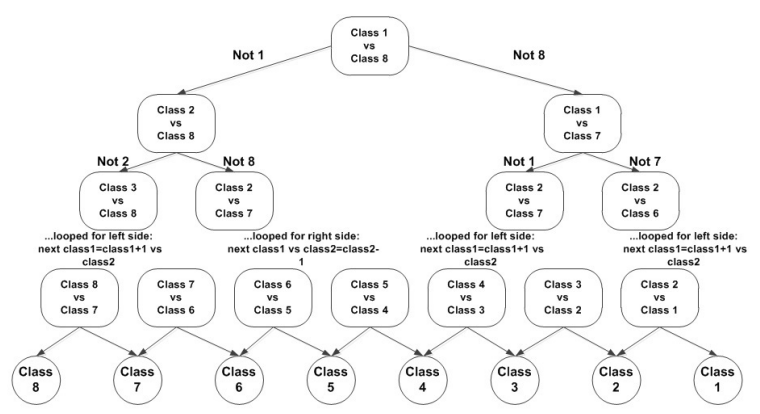

Fig. 8. DAG Decision Tree Method
TABLE I

ELM PERFORMANCE COMPARISON - All Classes IN \% ACCURACY, PRECISION, RECALL, AND TRAINING CPUTIME (Pose Cluster=35;Hidden Node Number 10X35)

\begin{tabular}{|l|c|c|c|c|}
\hline ELM Classifier & Accuracy & Precision & Recall & Time \\
\hline Single & 60.27 & 76.98 & 59.42 & $0.4992 \mathrm{~s}$ \\
\hline OAA & 56.26 & 79.50 & 49.42 & $3.9936 \mathrm{~s}$ \\
\hline OAO & 82.37 & 78.58 & 75.19 & $4.8672 \mathrm{~s}$ \\
\hline Binary-tree & 80.254 & 72.975 & 71.124 & $2.1216 \mathrm{~s}$ \\
\hline OAO-3Tree & 81.317 & 76.876 & 72.196 & $2.8392 \mathrm{~s}$ \\
\hline DAG & 88.80 & 91.76 & 89.02 & $4.8672 \mathrm{~s}$ \\
\hline
\end{tabular}

TABLE II

SVM PERFormance COMPARISON - All Classes in \% ACCURACY, PRECISION, RECALL, AND TRAINING CPUTIME (POSE CLUSTER $=35$; MAXITER $=1000)$

\begin{tabular}{|l|c|c|c|c|}
\hline SVM Classifier & Accuracy & Precision & Recall & Time \\
\hline OAA & 94.60 & 95.08 & 94.73 & $110.98 \mathrm{~s}$ \\
\hline OAO & 93.59 & 86.77 & 85.07 & $11.95 \mathrm{~s}$ \\
\hline DAG & 79.59 & 81.08 & 79.85 & $14.165 \mathrm{~s}$ \\
\hline
\end{tabular}

TABLE III

ELM PERFORMANCE - EACH Class ACCURACY COMPARISON IN \% (Pose Cluster $=35$; Hidden NOdE 10X35)

\begin{tabular}{|l|c|c|c|c|}
\hline ELM Classifier & Class 1 & Class 2 & Class 3 & Class 4 \\
\hline Single & 72.5 & 86.786 & 38.929 & 56.071 \\
\hline OAA & 62.5 & 86.786 & 16.071 & 50.357 \\
\hline OAO & 87.5 & 100 & $\mathbf{7 0 . 3 5 7}$ & 94.643 \\
\hline Binary-tree & 85 & 94.643 & $\mathbf{6 2 . 5}$ & 83.214 \\
\hline OAO-3Tree & 90 & 94.643 & $\mathbf{5 9 . 6 4 3}$ & 85.714 \\
\hline DAG & 100 & 100 & $\mathbf{7 2 . 1 4 3}$ & 75 \\
\hline ELM Classifier & Class 5 & Class 6 & Class 7 & Class 8 \\
\hline Single & 44.286 & 68.929 & 36.786 & 38.095 \\
\hline OAA & 29.643 & 70.714 & 42.143 & 35.238 \\
\hline OAO & 62.5 & 83.929 & $\mathbf{9 4 . 2 8 6}$ & $\mathbf{9 3 . 3 3 3}$ \\
\hline Binary-tree & 61.429 & 84.286 & $\mathbf{8 8 . 5 7 1}$ & $\mathbf{8 0 . 4 7 6}$ \\
\hline OAO-3Tree & 62.5 & 81.429 & $\mathbf{9 2 . 5}$ & $\mathbf{8 3 . 3 3 3}$ \\
\hline DAG & 37.5 & 100 & $\mathbf{8 1 . 0 7 1}$ & $\mathbf{1 0 0}$ \\
\hline
\end{tabular}

Hierarchical strategies like binary tree ELM, and OAO-3Tier ELM, have potential unequal classification treatment issue when the class quantity is even or odd quantity. OAO-3Tier ELM needs to group the class to be 3 class member and has issue to determine what become lonely class when the class quantity is even (See Fig. 7). Difference with SVM as binary classifier, ELM can be used as single classifier for multiclass situation directly to overcome lonely class issue. However, it still has potential unequal classification treatment issue that probably affected to the accuracy performance. But, ELM DAG with more efficient looping code has equal classification treatment.

\section{CONCLUSION}

In this paper, We have compared various multiclass ELM strategy for complex posed-based 3D human motion classification problems with irregular data. We found ELM OAO method with 3tree strategy in 


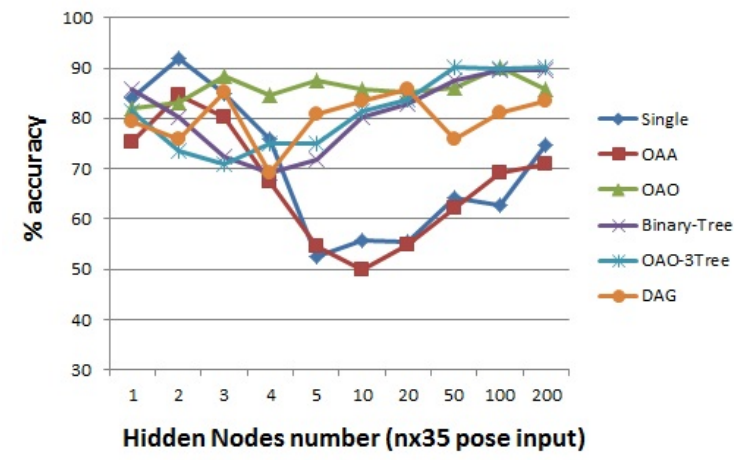

Fig. 9. ELM Accuracy performance for different hidden nodes\# (nx35 pose input)

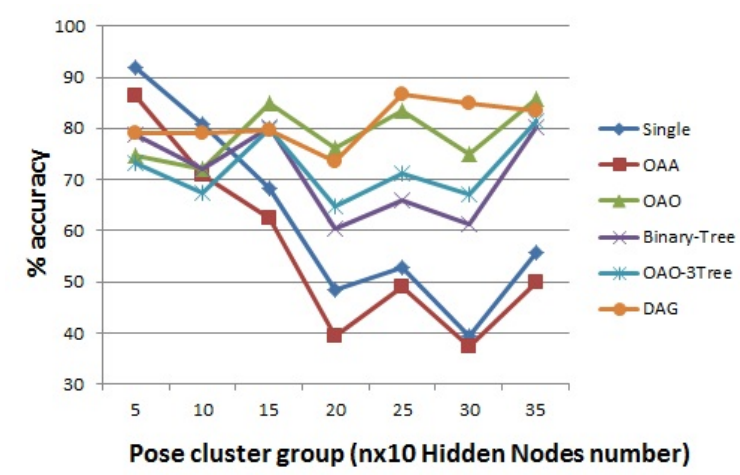

Fig. 10. ELM Accuracy performance for different pose cluster group (nx10 Hidden Nodes\#)

cascaded composition using Max-Win vote fusion of labeled output function gives the best result with accuracy approaching the classic OAO and DAG method but using less classifiers than OAO method. The ELM accuracy performance method is a bit lower than SVM methods but faster in training processing time. However, ELM OAO-3tree and Binary-tree have potential unequal classification treatment issue that probably affected to the accuracy performance. For our future research, we will do more various ELM MCS methods to compare with SVM MCS methods as our benchmark to get a better ELM multi classifier

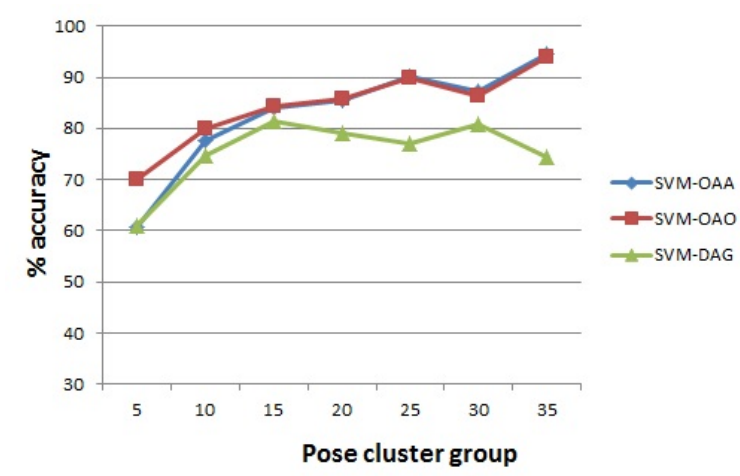

Fig. 11. SVM Accuracy performance for different pose cluster group strategies in terms of recognition accuracy and optimum structure for faster processing time for a realtime video recognition. ELM as eager reputation can handle complex multiclass classification problem without becoming fat by using MCS methods. However, determining a suitable classifier for a given problem is still more an art than a science (considering to no-freelunch theorem) and that is the contribution of MCS method to determine a suitable combination methods, rather than modifying single classifier only.

\section{ACKNOWLEDGMENT}

The Ministry of Research and Technology, Republic of Indonesia, funded this work under the National Synergy Research Grant SINas, contract No. RT-20121117

\section{REFERENCES}

[1] C.-Wei Hsu, C.-Jen Lin, "A comparison of methods for multiclass support vector machines," Neural Networks, IEEE Trans. on , vol.13, no.2, pp.415,425, 2002.

[2] F. Roli, G. Giacinto, G. Vernazza, "Methods for Designing Multiple Classifier Systems," Multiple Classifier Systems Lecture Notes in Computer Science, Vol. 2096, pp 78-87, 2001.

[3] G.-B Huang, H. Zhou; X. Ding, R. Zhang, "Extreme Learning Machine for Regression and Multiclass Classification," Systems, Man, and Cybernetics, Part B: Cybernetics, IEEE Trans. on, vol.42, no.2, pp.513,529, 2012.

[4] G.-B. Huang, D. H. Wang, and Y. Lan, "Extreme learning machines: a survey," International Journal of Machine Learning and Cybernetics, vol. 2, no. 2, pp. 107-122, 2011.

[5] G.-B. Huang, Q.-Y. Zhu, and C.-K. Siew, "Extreme learning machine: Theory and applications," Neurocomputing, vol. 70, pp. 489-501, 2006.

[6] G. Madzarov, D. Gjorgjevikj, "Multi-class classification using support vector machines in decision tree architecture," EUROCON 2009, pp.288-295, 2009.

[7] H.-J. Rong, G.-B. Huang, and Y.-S. Ong, "Extreme learning machine for multi-categories classification applications," Neural Networks, IJCNN, pp. 1709-1713, 2008.

[8] Kuncheva, L.I., "Combining Pattern Classifiers. In: Methods and Algorithms," Wiley, Chichester, 2004.

[9] M. Raptis, D. Kirovskiče, and H. Hoppe, "Real-time classification of dance gestures from skeleton animation," $A C M$ SIGGRAPH Symposium on Computer Animation, 2011.

[10] P. Ranganathan, A. Ramanan, M. Niranjan, "An efficient and speeded-up tree for multi-class classification," ICIAfS IEEE 6th Intl. Conference on , pp.190-193, 2012.

[11] R. Poppe, "A survey on vision-based human action recognition," ELSEVIER Image and Vision Computing, vol. 28, pp. 976-990, 2010.

[12] S. Abe, "Analysis of Multiclass Support Vector Machines", CIMCA, pp. 385-396, 2003.

[13] U.H.G. Kre $\beta$ el, "Pairwise classification and support vector machines," In B. Scholkopf, C. J. C. Burges, and A. J. Smola (Eds.), Advances in kernel methods: Support vector learning, pp. 255-268, Cambridge, MA: MIT Press, 1999.

[14] V.N. Vapnik, "The Nature of Statistical Learning Theory," Springer, New York, ISBN 0-387-98780-0, 2000.

[15] W. H. Liang Wang and T. Tan, "Recent development in human motion analysis," ELSEVIER Pattern Recognition, vol. 36, pp. 585-601, 2003.

[16] X. Wu, V. Kumar, J.R. Quinlan, J. Ghosh, Q. Yang, H. Motoda, G.J. McLachlan, A. Ng, B. Liu, P.S. Yu, Z.Zhou, M. Steinbach, D.J. Hand, D. Steinberg, "Top 10 algorithms in data mining: Survey Paper," Journal Knowledge and Information Systems, vol. 14:137, 2008.

[17] Y. Heryadi, M. Fanany, and A. Arymurthy, "A syntactical modeling and classification for performance evaluation of bali traditional dance," ICACSIS, pp. 261-265, 2012. 